\title{
Prevalência de Deficiência e Insuficiência de Vitamina $D$ e sua Correlação com PTH, Marcadores de Remodelação Óssea e Densidade Mineral Óssea, em Pacientes Ambulatoriais
}

\section{artigo originall}

bárbara C. Carvalho Silva

Bruno Muzzi Camargos

JuLIENNE Borges FuJII

EdUARdo Pimentel Dias

Maria Marta Sarquis Soares

Ambulatório de Doenças

Osteometabólicas do Serviço de

Endocrinologia da Santa Casa

de Belo Horizonte, MG, Brasil (BCCS); Densimater - Serviço de Densitometria Óssea do Hospital Mater Dei, Belo Horizonte, MG,

Brasil (BMC); Felicoop - Núcleo de Apoio à Pesquisa, Belo Horizonte, MG, Brasil (JBF);

Serviço de Endocrinologia do

Hospital Felício Rocho, Belo

Horizonte, MG, Brasil (EPD);

Instituto Felício Rocho de

Pesquisa e Educação

Continuada (Iferpec), MG, Brasil (EPD, MMSS); Departamento de

Clínica Médica da Universidade

Federal de Minas Gerais, Belo

Horizonte, MG, Brasil (MMSS).

Recebido em 26/06/2007

Aceito em 03/12/2007

\section{RESUMO}

A deficiência de vitamina $D$ (VD) leva ao raquitismo e à osteomalacia e sua insuficiência, caracterizada pelo hiperparatireoidismo secundário, pode resultar osteoporose. Apesar de amplamente prevalente, a insuficiência de VD ainda é pouco reconhecida e subtratada. Os autores estudaram 180 pacientes atendidos em ambulatório de endocrinologia em Belo Horizonte, que tiveram os níveis de 25(OH)VD mensurados, correlacionando-os com paratormônio (PTH), marcadores de remodelação óssea e densidade mineral óssea. Para caracterização de níveis insuficientes de VD, foram correlacionados os níveis de 25(OH)VD com os de PTH, definindo-se, nesta série, ponto de corte de $25(\mathrm{OH}) \mathrm{VD}$ de $32 \mathrm{ng} / \mathrm{ml}$. Foi encontrada correlação inversa e significativa entre 25(OH)VD e PTH e entre 25(OH)VD e C-telopeptídeo. A prevalência de insuficiência de VD na população estudada foi de $42,4 \%$. Conclui-se que a insuficiência de VD tem alta prevalência entre pacientes que freqüentam nossos consultórios, alertando para a importância da sua investigação na prática clínica e na instituição de políticas para sua prevenção. (Arq Bras Endocrinol Metab 2008; 52/3:482-488)

Palavras-chave: Vitamina D; Paratormônio; Marcadores de remodelação óssea; Densidade mineral óssea; Insuficiência de vitamina D

\section{ABSTRACT}

Prevalence of Vitamin D Deficiency and its Correlation with PTH, Biochemical Bone Turnover Markers and Bone Mineral Density, Among Patients from Ambulatories.

Consequences of VD deficiency include rickets and osteomalacia. However, marginal concentrations of 25-hydroxyvitamin D (25(OH)VD) are associated with secondary hyperparathyroidism and osteoporosis. In this context, levels of $25(\mathrm{OH}) \mathrm{VD}$ capable to elevate parathyroid hormone (PTH) could be considered as insufficient. The VD insufficiency, although widely prevalent, is still under-recognized and under-treated. The authors have studied 180 patients followed in a endocrinology clinic in Belo Horizonte, who had 25(OH)VD measured, correlating it with PTH, biochemical bone turnover markers and bone mineral density. To determine the sufficiency of VD, 25(OH)VD was correlated with PTH and the cutoff found was of $32 \mathrm{ng} / \mathrm{ml}$. CTX-1 and PTH were significantly negative correlated to $25(\mathrm{OH}) \mathrm{VD}$ and the prevalence of VD insufficiency was $42 \%$. One concludes that the VD insufficiency is widely prevalent among patients who frequently come to our offices, alerting us for the importance to assess VD status more often and to practice politics for prevention of VD insufficiency. (Arq Bras Endocrinol Metab 2008; 52/3:482-488)

Key words: Vitamin D; Parathyroid hormone; Bone turnover markers; Bone mineral density; Vitamin D insufficiency 


\section{INTRODUÇÃO}

VITAMINA D (VD), considerada um hormônio esteróide desde meados da década de 1960 (1), é de fundamental importância para a homeostase do cálcio e do fósforo e para a saúde musculoesquelética (2). Além disso, níveis insuficientes de VD vêm sendo implicados na patogênese e na progressão de várias outras desordens, incluindo doenças cardiovasculares, câncer de cólon e próstata, esclerose múltipla, diabetes melito tipos 1 e 2, doença inflamatória intestinal, entre outras (2-11).

A vitamina $\mathrm{D}_{3}$ ou colecalciferol é sintetizada na pele humana pela ação da radiação UV-B a partir do 7-dehidrocolesterol, além de ser encontrada em certos alimentos, como óleo de peixe e gema de ovo $(1,12)$. A vitamina $\mathrm{D}_{2}$ ou ergocalciferol é formada a partir de um esteróide fúngico, o ergosterol, sendo pouco disponível naturalmente nos alimentos, mas usada como suplemento alimentar (12). Uma vez ingerida ou sintetizada na pele, a VD é transportada até o fígado, onde sofre a primeira hidroxilação no carbono 25 , convertendo-se a 25 hidroxi-vitamina D $(25(\mathrm{OH}) \mathrm{VD})$. A $25(\mathrm{OH}) \mathrm{VD}$ é a principal forma de VD circulante, com meia-vida em torno de duas a três semanas. Sua concentração sérica tem estreita correlação com os achados de osteomalacia e raquitismo e com a produção cutânea ou ingesta de VD, refletindo de maneira segura os estoques corporais desta vitamina $(1,7,12-14)$. No rim, a $25(\mathrm{OH})$ VD sofre nova hidroxilação com a produção de sua forma ativa, a 1,25 dihidroxi-vitamina $\mathrm{D}\left(1,25(\mathrm{OH})_{2} \mathrm{D}\right)$. Apesar de se tratar da forma ativa, os níveis séricos de $1,25(\mathrm{OH})_{2} \mathrm{D}$ não se prestam para uma estimativa do estoque corporal de VD, pois podem estar normais na osteomalacia leve ou moderada, em decorrência do hiperparatireoidismo secundário que acompanha esta condição. Além disso, a $1,25(\mathrm{OH})_{2} \mathrm{D}$ tem meia-vida menor, em torno de seis a oito horas, e sua produção local, como a que ocorre em várias células do organismo, não pode ser estimada pelos níveis circulantes deste metabólito (13).

A deficiência de VD prejudica a mineralização óssea, levando ao raquitismo e à osteomalacia, em crianças, e à osteomalacia, nos adultos $(7,12)$. No entanto, reduções menos graves nos níveis de VD, com concentrações séricas de $25(\mathrm{OH}) \mathrm{VD}$ dentro dos valores de referência normais, podem levar à redução na calcemia, resultando hiperparatireoidismo secundário, perda óssea e osteoporose (1,2,7,13-17). Nesse contexto, níveis de $25(\mathrm{OH})$ VD abaixo dos quais começa haver alteração na homeostase do cálcio e elevação do PTH, carac- terizando o hiperparatireoidismo secundário, poderiam ser considerados como insuficientes $(1,14,18,19)$. Muitos autores consideram níveis séricos insuficientes de $25(\mathrm{OH}) \mathrm{VD}$, aqueles abaixo de 30 a $32 \mathrm{ng} / \mathrm{ml}(7,14,17$ 19). No entanto, há grande variabilidade entre os ensaios de diferentes laboratórios, dificultando a determinação de um nível ideal universal de $25(\mathrm{OH}) \mathrm{VD}$, e alguns autores sugerem que seria mais prudente a determinação de níveis normais de $25(\mathrm{OH}) \mathrm{VD}$ para cada população, para cada método e laboratório $(1,14,18)$. Essa determinação faz-se importante uma vez que a insuficiência de VD parece ser freqüente em nosso meio, mas ainda pouco reconhecida e, portanto, subtratada (14).

Fundamentados nisso, os autores estudaram uma população de pacientes residentes na região metropolitana de Belo Horizonte-MG com endocrinopatias variadas, com o objetivo de identificar a prevalência de deficiência de VD, correlacionando concentrações séricas de $25(\mathrm{OH})$ VD com níveis de PTH, marcadores de remodelação óssea e densitometria óssea da coluna lombar e do fềmur.

\section{PACIENTES E MÉTODOS}

Por meio de revisão de prontuários, foram selecionados 180 pacientes acompanhados em ambulatório de endocrinologia, por diferentes motivos, que tivessem tido os níveis séricos de $25(\mathrm{OH}) \mathrm{VD}$ mensurados, em um mesmo laboratório, ao longo de todo o ano de 2006. Quando disponíveis, exames de densitometria óssea e dosagens séricas de PTH intacto (PTH), cálcio total corrigido pela albumina e/ou cálcio iônico, C-telopeptídeo (CTX-1) e fosfatase alcalina óssea (FAO) foram utilizados para a análise dos dados. Foram excluídos os pacientes com alteração da função renal ou hepática e aqueles em uso de medicações que, sabidamente, interferem no metabolismo da VD. Os pacientes em uso de suplemento de VD foram incluídos na análise. Todos os pacientes eram provenientes da cidade de Belo Horizonte, MG, que está na latitude de $19^{\circ} \mathrm{S}$.

Para realização da correlação entre os níveis de 25(OH)VD com níveis de PTH, CTX-1, FAO, calcemia e densidade mineral óssea, foram excluídos os pacientes com diagnóstico de hiperparatireoidismo primário, hiper ou hipotireoidismo não tratados e aqueles em uso de bisfosfonatos, terapia de reposição hormonal, modulador seletivo do receptor estrogênico, 
corticóide, suplementos de cálcio e VD e outros medicamentos capazes de influenciar o metabolismo do cálcio e a remodelação óssea.

A $25(\mathrm{OH}) \mathrm{VD}$ foi dosada por HPLC com coeficiente de variação interensaio de $10 \%$ e valor de referência de 14 a $80 \mathrm{ng} / \mathrm{ml}$. O PTH foi dosado por quimioluminescência, com coeficiente de variação interensaio de $7 \%$ e valor de referência de 7 a $53 \mathrm{pg} / \mathrm{ml}$. O CTX-1 foi dosado no soro por eletroquimioluminescência com limite superior da normalidade, em homens abaixo de 50 anos e em mulheres na pré-menopausa, de até 0,584 e 0,573 nanog $/ \mathrm{ml}$, respectivamente. O coeficiente de variação interensaio do CTX-1 varia de $2,10 \%$ a $3,56 \%$. A FAO foi dosada pelo método de imunoensaio por captura, com coeficiente de variação interensaio de $5,2 \%$ e valores de referência entre 11,6 a $30,6 \mathrm{U} / \mathrm{L}$ para mulheres pré-menopausadas e 15 a 41,3 U/L, em homens.

As densitometrias ósseas foram realizadas em dois serviços, acreditados pela Sociedade Brasileira de Densitometria (SBDENS), que utilizam o mesmo recurso tecnológico: densitômetro fan-beam da marca GE/Lunar, modelo Prodigy Advanced Plus, com software versão 9.3 , de setembro de 2005. Cada paciente foi submetido a um único exame, com finalidade diagnóstica, dispensando-se, com isso, exames cruzados de phantom, considerados ideais quando exames seriados são realizados. Foram adquiridos exames de coluna e fềmur. Todos os exames atenderam às recomendações técnicas vigentes à época, de acordo com as posições oficiais preconizadas pela International Society for Clinical Densitometry (ICSD).

A análise estatística dos dados foi desenvolvida utilizando técnicas descritivas e exploratórias (média, mediana, desvio-padrão, tabelas de freqüências e gráficos), testes comparativos não-paramétricos (Mann-Whitney e Kruskal-Wallis) e análise de correlação (Spearman). O nível de significância adotado foi de $5 \%(\alpha=0,05)$.

\section{RESULTADOS}

Foram avaliados 180 pacientes, sendo $165(91,6 \%)$ do sexo feminino. A idade variou entre 14 a 91 anos, com média de 58,87 anos. Os diagnósticos mais freqüentemente encontrados foram hipotireoidismo em $27,3 \%$ dos casos, osteoporose em $26,3 \%$, diabetes melito tipo $2 \mathrm{em}$ $13,4 \%$, climatério em $12,9 \%$, hipertensão em $9,1 \%$, dislipidemia em $5,3 \%$ e hiperparatireoidismo primário em 2,9\%
Entre os pacientes avaliados, independentemente do diagnóstico, incluindo aqueles em uso de suplementos de $\mathrm{VD}$, os valores de $25(\mathrm{OH}) \mathrm{VD}$, em ng/ml, variaram entre 11 e 80,3 , com média $( \pm \mathrm{DP})$ de $39,79 \pm 16,76$ e mediana de 36,45 .

Excluindo-se os pacientes em uso de suplementos de VD, obteve total de 132 pacientes. Neste grupo, os valores de $25(\mathrm{OH}) \mathrm{VD}$, em ng/ml, variaram de 11,0 a 76,3 , com média $( \pm \mathrm{DP})$ de $38,23 \pm 15,62$ e mediana de 34,30 . Considerando níveis deficientes e insuficientes de VD aqueles abaixo de $14 \mathrm{ng} / \mathrm{ml}$ e $32 \mathrm{ng} / \mathrm{ml}$, respectivamente, $0,8 \%$ destes pacientes tinham deficiência e $42,4 \%$ tinham insuficiência de VD.

No grupo que estava em uso de suplementos de $\mathrm{VD}$, representado pelo total de 48 pacientes, não houve casos de deficiência de VD [25(OH)VD abaixo de $14 \mathrm{ng} / \mathrm{ml}$ ], mas 13 pacientes (27\%) tinham níveis insuficientes de VD [25(OH)VD abaixo de $32 \mathrm{ng} / \mathrm{ml}$ ]. A porcentagem de pacientes com insuficiência de VD foi menor entre o grupo em reposição de VD; esta diferença foi estatisticamente significativa $(\mathrm{p}=0,047)$.

Comparando a VD em relação ao sexo e à faixa etária, em anos, não foi identificada diferença significativa, com valor $\mathrm{p}>0,05$ para ambas as comparações.

Depois de exclusões pertinentes, correlacionou-se níveis de $25(\mathrm{OH}) \mathrm{VD}$ com níveis de PTH (56 pacientes), CTX-1 (51 pacientes), FAO (52 pacientes), DMO (58 pacientes) e calcemia (86 pacientes). Houve correlação significativa e negativa entre os níveis de $25(\mathrm{OH}) \mathrm{VD}$ e PTH $(r=-0,416$ e valor $\mathrm{p}=0,001)$ e entre $25(\mathrm{OH}) \mathrm{VD}$ e CTX-1 $(\mathrm{r}=-0,292$ e valor $\mathrm{p}=0,038)$, representadas nas Figuras 1 e 2, respectivamente. Não houve correlação estatisticamente significativa entre $25(\mathrm{OH}) \mathrm{VD}$ e densidade mineral óssea ou calcemia. Também não houve correlação significativa entre $25(\mathrm{OH}) \mathrm{VD}$ e FAO, apesar de ter sido observada tendência a níveis elevados de FAO entre os pacientes com níveis menores de $25(\mathrm{OH}) \mathrm{VD}$.

Considerando o limite máximo da normalidade para PTH de $53 \mathrm{pg} / \mathrm{ml}$, foram verificados valores de $25(\mathrm{OH})$ VD significativamente mais baixos entre os pacientes com PTH acima do valor de referência $(\mathrm{p}=0,001)$ (Figura 3 ). Avaliando possíveis pontos de corte para caracterizar insuficiência de VD na nossa série, a fim de identificar um ponto abaixo do qual começa ocorrer aumento do PTH, obteve-se a melhor correlação com níveis de $25(\mathrm{OH}) \mathrm{VD}$ de $32 \mathrm{ng} / \mathrm{ml}(\mathrm{p}=0,000)$ (Figura 1$)$. Com níveis de 25(OH)VD acima de $32 \mathrm{ng} / \mathrm{ml}$, o PTH atinge platô, com concentrações aproximadas de $40 \mathrm{pg} / \mathrm{ml}$. 


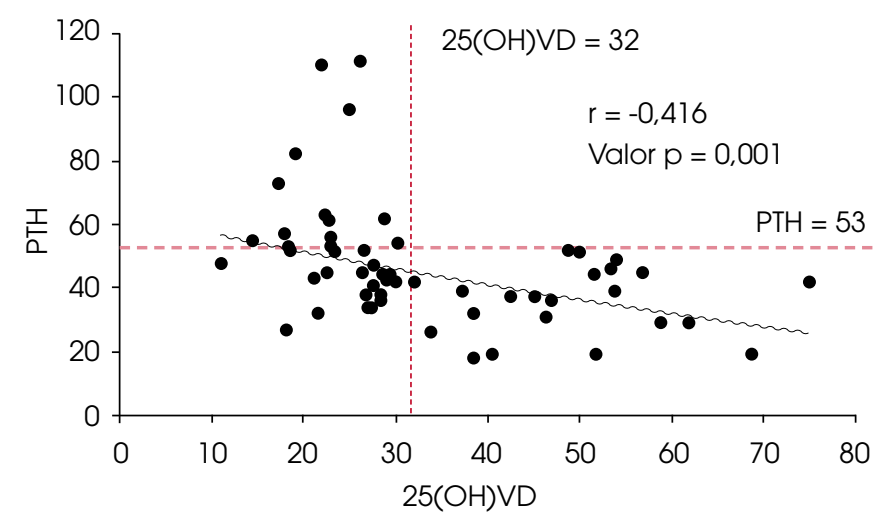

Figura 1. Correlação entre 25(OH)VD e PTH.

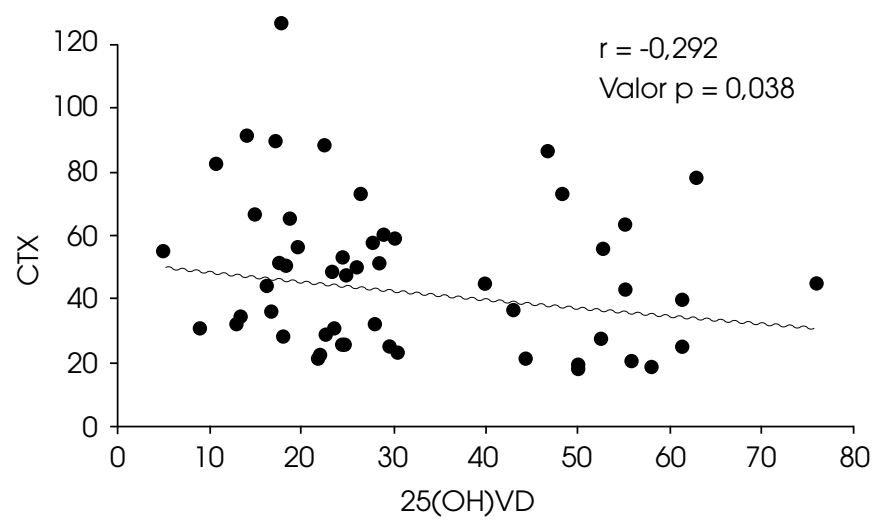

Figura 2. Correlação entre 25(OH)VD e CTX-1.

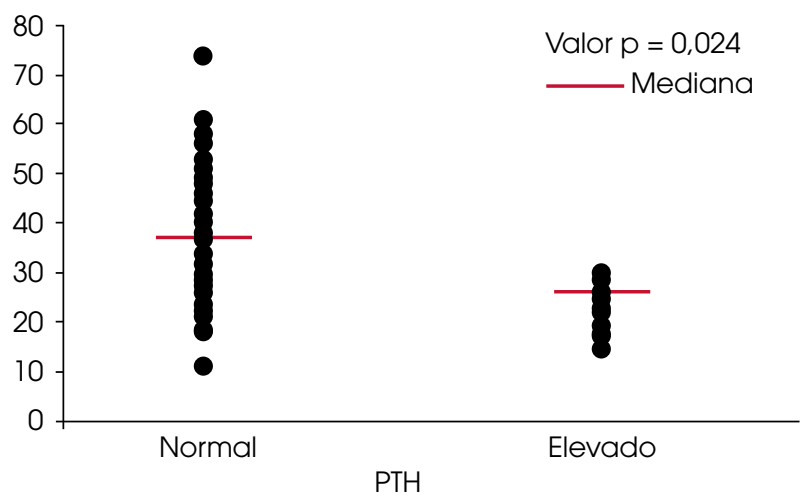

Figura 3. Comparação entre concentrações de 25(OH)VD em pacientes com PTH normal e elevado.

\section{DISCUSSÃo}

A insuficiência de VD constitui hoje uma epidemia não reconhecida em várias populações de todo o mundo (2). Sabe-se que níveis séricos de $25(\mathrm{OH}) \mathrm{VD}$, ditos normais, nem sempre refletem concentrações suficientes para manutenção da saúde óssea e muscular, podendo inclusive aumentar o risco de doenças não osteomusculares, como neoplasias ou doenças inflamatórias e cardiovasculares (2-11).

Para a determinação de níveis ótimos de $25(\mathrm{OH})$ $\mathrm{VD}$, o ideal seria correlacionar sua concentração sérica com eventos hipoteticamente relacionados à sua insuficiência. Um estudo com este desenho, no entanto, é desafiador, uma vez que doenças ligadas à insuficiência de VD são, em sua maioria, crônicas, com longo período de latência e com causas multifatoriais (20).

Alternativamente, alguns autores fundamentam-se nos níveis de PTH para estabelecer níveis satisfatórios de VD. Os limites de concentração sérica de $25(\mathrm{OH})$ $\mathrm{VD}$, que separam a suficiência da insuficiência desta vitamina, poderiam ser definidos por seus efeitos biológicos conhecidos no metabolismo ósseo e mineral, refletidos, particularmente, no aumento do PTH (19). Assim, valores de VD abaixo dos quais ocorre elevação dos níveis de PTH, caracterizando uma alteração na homeostase do cálcio e hiperparatireoidismo secundário, seriam indicativos de insuficiência de VD.

Neste trabalho, os autores encontraram aumento do PTH quando os níveis de $25(\mathrm{OH}) \mathrm{VD}$ estão abaixo de $32 \mathrm{ng} / \mathrm{ml}$. Considerando como insuficientes os níveis de $25(\mathrm{OH}) \mathrm{VD}$ abaixo deste valor, demonstrou-se a prevalência de insuficiência de VD de $42,4 \%$ entre os pacientes que não faziam uso de suplementos de VD. No grupo de usuários destes suplementos, com apresentações e doses variadas, $27 \%$ dos pacientes ainda tinham níveis insuficientes desta vitamina. Comparando os dois grupos, constatou-se que esta diferença é estatisticamente significativa. Além disso, houve correlação inversa e significativa entre PTH e 25(OH)VD $(r=-0,416$ e $\mathrm{p}=0,001)$, nos dois grupos analisados.

Binkley e cols. (18) encontraram valores discordantes de 25(OH)VD obtidos de diferentes laboratórios, e Bandeira e cols. (14) sugerem que níveis normais de 25(OH)VD devem ser estabelecidos em cada região. De acordo com o conhecimento dos autores, este é o primeiro estudo em Belo Horizonte que avaliou níveis de VD e sua correlação com PTH, marcadores de remodelação e densidade mineral ós- 
sea. Todos os pacientes incluídos tiveram 25(OH)VD mensurada por HPLC, método padrão-ouro, em um mesmo laboratório. Os níveis de VD considerados suficientes nesta série foram semelhantes aos encontrados na literatura (14,19,21-23). Da mesma forma, a alta prevalência de insuficiência de VD demonstrada, foi verificada em outros trabalhos envolvendo crianças, adolescentes, adultos e idosos, saudáveis ou com doenças osteomusculares, institucionalizados ou não $(2,12,14,19,23-27)$. Apesar deste estudo ter sido conduzido em uma cidade ensolarada, situada a baixa latitude $\left(19^{\circ} \mathrm{S}\right)$, isso não foi o bastante para prevenir a alta prevalência de insuficiência de VD demonstrada. Outros autores também encontraram baixos níveis de $25(\mathrm{OH}) \mathrm{VD}$ em indivíduos sadios e ativos vivendo em regiões com ampla disponibilidade de luz solar $(28,29)$.

As causas para a grande prevalência de insuficiência de VD, mesmo em regiões de baixa latitude, são várias. A síntese de VD é proporcional à área exposta à luz solar e sofre influência de fatores ambientais, como latitude, estação do ano, hora do dia, quantidade de nuvens ou camada de ozônio, e de fatores relacionados ao próprio indivíduo e aos seus hábitos e costumes $(17,30)$. Tipo de pele (quanto mais melanina, menor a quantidade de VD sintetizada para uma mesma dose de UV-B), uso de protetor solar (uso correto de protetor solar com FPS 8 ou 15 reduz a capacidade de síntese de VD em 95\% e 99,9\%, respectivamente), idade (idosos têm quantidade reduzida de 7-dehidrocolesterol, reduzindo a capacidade de síntese de VD) e uso de roupas que cubram grande parte do corpo são fatores que influenciam a síntese cutânea de VD $(30,31)$. Atualmente, grande parte dos habitantes de centros urbanos desenvolvidos não mais se expõe à quantidade suficiente de luz solar para satisfazer as necessidades biológicas de VD (4).
A ingestão de VD é baixa, uma vez que sua ocorrência natural nos alimentos é pequena e a suplementação de alimentos com esta vitamina não é feita de modo universal $(4,17,32)$. Para Vieth (17), sem exposição aos raios UV-B, a quantidade de VD obtida de fontes alimentares e suplementos disponíveis nos dias atuais é insuficiente para manter a concentração sérica de 25( $\mathrm{OH}) \mathrm{VD}$ acima de $30 \mathrm{ng} / \mathrm{ml}$, em adultos.

Uma metanálise publicada recentemente demonstrou que o uso de doses diárias de 700 a 800 UI de colecalciferol diminui o risco de fraturas em idosos (33). A redução do risco de fraturas poderia ser explicada por redução da perda de massa óssea e melhora da força muscular em membros inferiores, reduzindo o número de quedas, sobretudo em idosos $(15,34,35)$. Evidências apontam para uma associação positiva entre os níveis de $25(\mathrm{OH}) \mathrm{VD}$ e densidade mineral óssea em fêmur e coluna lombar $(36,37)$. Apesar disso, esta correlação não foi significativa nesta série, talvez por ter sido feita em um número pequeno de casos. Da mesma forma, a correlação entre $25(\mathrm{OH}) \mathrm{VD}$ e $\mathrm{FAO}$ não foi significativa, apesar da demonstração, por outros autores, de uma correlação negativa entre estas variáveis (16). Por outro lado, houve relação negativa e significativa entre o marcador de reabsorção óssea CTX-1 e $25(\mathrm{OH}) \mathrm{VD}(\mathrm{r}=-0,292$ e valor $\mathrm{p}=0,038)$.

Os níveis de VD definidos como suficientes nessa casuística, com base na elevação do PTH sérico, foram aqueles superiores a $32 \mathrm{ng} / \mathrm{ml}$. Este valor deve ser individualizado, pois sabe-se que alguns indivíduos podem desenvolver hiperparatireoidismo secundário com níveis de $25(\mathrm{OH}) \mathrm{VD}$ mais altos $(22,38)$. Além disso, autores que avaliaram eventos relacionados à insuficiência de VD constataram a necessidade de níveis mais elevados de $25(\mathrm{OH}) \mathrm{VD}$ para a prevenção de outras doenças (Tabela 1 ).

Tabela 1. Níveis de 25(OH)VD para prevenção de doenças relacionadas à insuficiência de VD.

\begin{tabular}{|c|c|c|}
\hline Doença & Concentração de 25(OH)VD & Referência \\
\hline Esclerose múltipla & $100 \mathrm{nmol} / \mathrm{l}(40 \mathrm{ng} / \mathrm{ml})$ & \multirow[t]{2}{*}{10 (estudo caso-controle prospectivo) } \\
\hline Fraturas ósseas & $100 \mathrm{nmol} / \mathrm{l}(40 \mathrm{ng} / \mathrm{ml})$ & \\
\hline Doença periodontal & 90 a $100 \mathrm{nmol} / \mathrm{l}(36$ a 40 ng/ml) & \multirow{3}{*}{$\begin{array}{l}15 \text { (metanálise de estudos controlados, } \\
\text { randomizados e duplos-cegos) }\end{array}$} \\
\hline Câncer de cólon & $90 \mathrm{nmol} / \mathrm{l}(36 \mathrm{ng} / \mathrm{ml})$ & \\
\hline $\begin{array}{l}\text { Fraqueza muscular com maior } \\
\text { propensão a quedas }\end{array}$ & $\begin{array}{c}40 \mathrm{nmol} / \mathrm{l}(16 \mathrm{ng} / \mathrm{ml}) \text { ou, idealmente, } \\
90 \mathrm{a} 100 \mathrm{nmol} / \mathrm{l}(36 \text { a } 40 \mathrm{ng} / \mathrm{ml})\end{array}$ & \\
\hline Câncer de próstata & $40 \mathrm{nmol} / \mathrm{l}(16 \mathrm{ng} / \mathrm{ml})$ & 39 (estudo caso-controle prospectivo) \\
\hline
\end{tabular}


Não se avaliou quais seriam as doses ideais de reposição de VD para a manutenção de níveis satisfatórios de $25(\mathrm{OH}) \mathrm{VD}$. Em artigo de revisão publicado recentemente (40), Holick sugere que a reposição de VD para adultos ou idosos com exposição solar ou suplementação insuficientes seja feita com ergocalciferol (vitamina $\mathrm{D}_{2}$ ), na dose de 50.000 UI por semana, por 8 semanas. A dosagem de $25(\mathrm{OH}) \mathrm{VD}$ deve ser realizada ao término do tratamento e o mesmo esquema repetido caso seus níveis permaneçam inferiores a $30 \mathrm{ng} / \mathrm{ml}$. Se, por outro lado, este nível for atingido, sugere manter 50.000 UI de $\mathrm{D}_{2}$ a cada 15 ou 30 dias, para prevenção da deficiência. Holick e outros autores sugerem, ainda, como alternativa, doses de 800 a 1.000 UI por dia de colecalciferol $(1,15,40)$, mas não sabemos se esses esquemas terapêuticos são válidos para nossa população. Enquanto isso, acreditamos que o ideal seria a individualização da dose de reposição de VD ajustada por meio da monitorização dos níveis de calcemia, calciúria e $25(\mathrm{OH}) \mathrm{VD}$.

\section{CONCLUSÃO}

Existe alta prevalência de insuficiência de VD entre os pacientes avaliados e há uma correlação significativa e inversa entre $25(\mathrm{OH}) \mathrm{VD}$ e PTH e $25(\mathrm{OH}) \mathrm{VD}$ e CTX-1. Níveis de 25(OH)VD que melhor se correlacionaram com elevação do PTH sérico foram aqueles inferiores a $32 \mathrm{ng} / \mathrm{ml}$, embora níveis desta vitamina em torno de $40 \mathrm{ng} / \mathrm{ml}$ pareçam ser necessários para prevenção de outras doenças. Políticas de prevenção de insuficiência de VD, como a fortificação de alimentos com esta vitamina, deveriam ser adotadas, mesmo em países localizados em latitudes mais baixas. A avaliação dos níveis de $25(\mathrm{OH}) \mathrm{VD}$ deveria fazer parte da propedêutica médica habitual, uma vez que não há outra forma de mensurarmos os estoques de VD e adequá-los para cada paciente individualmente.

\section{REFERÊNCIAS}

1. Premaor MO, Furlanetto TW. Hipovitaminose D em adultos: entendendo melhor a apresentação de uma velha doença. Arq Bras Endocrinol Metab. 2006;50(1):25-37.

2. Holick MF. High prevalence of vitamin D inadequacy and implications for health. Mayo Clin Proc. 2006;81(3):353-73.

3. Zittermann A. Vitamin $D$ and disease prevention with special reference to cardiovascular disease. Prog Biophys Mol Biol. 2006:92:39-48
4. Holick MF. Vitamin D: its role in cancer prevention and treatment. Prog Biophys Mol Biol. 2006;92:49-59.

5. Cantorna MT. Vitamin D and its role in immunology: multiple sclerosis and inflammatory bowel disease. Prog Biophys Mol Biol. 2006;92:60-4.

6. Grant WB. Epidemiology of disease risks in relation to vitamin D insufficiency. Prog Biophys Mol Biol. 2006;92:65-79.

7. Holick MF. Resurrection of vitamin D deficiency and rickets. J Clin Invest. 2006;116:2062-72.

8. Mathieu C, Gysemans C, Giulietti A, Bouillon R. Vitamin D and diabetes. Diabetologia. 2005;48:1247-57.

9. Mathieu C, Badenhoop K. Vitamin D and type 1 diabetes mellitus: state of the art. Trends Endocrinol Metab. 2005;16:261-6.

10. Munger KL, Levin LI, Hollis BW, Howard NS, Ascherio A. Serum 25-hydroxyvitamin $D$ levels and risk of multiple sclerosis. JAMA. 2006;296:2832-8.

11. Pittas AG, Lau J, Hu FB, Dawson-Hughes B. The role of vitamin $D$ and calcium in type 2 diabetes. A systematic review and meta-analysis. J Clin Endocrinol Metab. 2007;92:2017-29.

12. Munns C, Zacharin MR, Rodda CP, Batch JA, Morley R, Cranswick NE, et al. Prevention and treatment of infant and childhood vitamin D deficiency in Australia and New Zealand: consensus statement. Med J Aust. 2006;185:268-72.

13. Mosekilde L. Vitamin D and the elderly. Clin Endocrinol. 2005;62(3):265-81

14. Bandeira F, Griz L, Dreyer P, Eufrazino C, Bandeira C, Freese E. Vitamin D deficiency: a global perspective. Arq Bras Endocrinol Metab. 2006;50(4):640-6.

15. Bischoff-Ferrari HA, Giovannucci E, Willett WC, Dietrich T, Dawson-Hughes B. Estimation of optimal serum concentrations of 25-hydroxyvitamin D for multiple health outcomes. Am J Clin Nutr. 2006;84:18-28.

16. Need AG. Bone resorption markers in vitamin $D$ insufficiency. Clin Chim Acta. 2006;368:48-52.

17. Vieth R. What is the optimal vitamin D status for health? Prog Biophys Mol Biol. 2006;92:26-32.

18. Binkley N, Krueger D, Cowgill CS, Plum L, Lake E, Hansen KE, et al. Assay variation confounds the diagnosis of hypovitaminosis D: a call for standardization. J Clin Endocrinol Metab. 2004:89:3152-7.

19. Chapuy MC, Preziosi P, Maamer M, Arnaud S, Galan P, Hercberg $S$, et al. Prevalence of vitamin $D$ insufficiency in an adult normal population. Osteoporos Int. 1997;7:439-43.

20. Heaney RP. Nutrition and chronic disease. Mayo Clin Proc. 2006;81(3):297-299.

21. Malabanan A, Veronikis IE, Holick MF. Redefinig vitamin D insufficiency. Lancet. 1998;351:805-6.

22. Dawson-Hughes B, Heaney RP, Holick MF, Lips P, Meunier PJ, Vieth R. Estimates of optimal vitamin D status. Osteoporos Int 2005;16:713-6.

23. Holick MF, Siris ES, Binkley N, Beard MK, Khan A, Katzer JT, et al. Prevalence of vitamin D inadequacy among postmenopausal north american women receiving osteoporosis therapy. $J$ Clin Endocrinol Metab. 2005:90:3215-24.

24. Armstrong DJ, Meenagh GK, Bickle I, Lee ASH, Curran ES, Finch MB. Vitamin D deficiency is associated with anxiety and depression in fibromyalgia. Clin Rheumatol. 2007;26:551-4.

25. Gregory A, Plotnikoff MTS, Quigley JM. Prevalence of severe hypovitaminosis $D$ in patients with persistent, nonspecific musculoskeletal pain. Mayo Clin Proc. 2003;78:1463-70. 
26. Gordon CM, Peter KC, Feldman HA, Grace E, Emans J. Prevalence of vitamin $D$ deficiency among healthy adolescents. Arch Pediatr Adolesc Med. 2004;158:531-7.

27. Saraiva GL, Cendoroglo MS, Ramos LR, Araújo LMQ, Vieira JGH, Maeda SS, et al. Prevalência da deficiência, insuficiência de vitamina $\mathrm{D}$ e hiperparatireoidismo secundário em idosos institucionalizados e moradores na comunidade da cidade de São Paulo, Brasil. Arq Bras Endocrinol Metabol. 2007;51:437-42.

28. Binkley N, Novotny R, Krueger D, Kawahara T, Daida YG, Lensmeyer G, et al. Low vitamin D status despite abundant sun exposure. J Clin Endocrinol Metabolism. 2007;92:2130-5.

29. Sedrani SH. Low 25-hydroxyvitamin D and normal serum calcium concentrations in Saudi Arabia: riyadh region. Ann Nutr Metab. 1984;28:181-5.

30. Webb AR. Who, what, where and when-influences on cutaneous vitamin D synthesis. Progr Biophys Mol Biol. 2006;92:17-25.

31. Holick MF. Vitamin D deficiency: what a pain it is? Mayo Clin Proc. 2003;78(12):1457-9.

32. Calvo MS, Whiting SJ, Barton CN. Vitamin D intake: a global perspective of current status. J Nutr. 2005;135:310-6.

33. Bischoff-Ferrari HA, Willett WC, Wong JB, Giovannucci E, Dietrich T, Dawson-Hughes B. Fracture prevention with vitamin $D$ supplementation. A neta-analysis of randomized controlled trials. JAMA. 2005;293:2257-64.

34. Wicherts IS, van Schoor NM, Boeke AJP, Visser M, Deeg DJH, Smit J, et al. Vitamin D status predicts physical performance and its decline in older persons. J Clin Endocrinol Metab. 2007;92:2058-65.
35. Heath KM, Elovic EP. Vitamin D deficiency - implications in the rehabilitation setting. Am J Phys Med Rehabil. 2006; 85:916-23.

36. Bischoff-Ferrari HA, Dietrich T, Orav EJ, Dawson-Hughes B. Positive association between 25-hydroxy vitamin D levels and bone mineral density: a population-based study of younger and older adults. Am J Med. 2004;116:634-9.

37. Mezquita-Raya P, Munoz-Torres M, Luna JD, et al. Relation between vitamin $D$ insufficiency, bone density, and bone metabolism in healthy postmenopausal women. J Bone Miner Res. 2001;16:1408-15.

38. Vieth R, Ladak Y, Walfish PG. Age-related changes in the 25hydroxyvitamin $D$ versus parathyroid hormone relationship suggest a different reason why older adults require more vitamin D. J Clin Endocrinol Metab. 2003;88:185-91.

39. Ahonen MH, Tenkanen L, Teppo L, Hakama M, Tuohimaa P. Prostate cancer risk and prediagnostic serum 25-hydroxyvitamin $D$ levels (Finland). Cancer Causes and Control. 2000;11:847-52.

40. Holick MF. Vitamina D deficiency. N Engl J Med. 2007; 357:266-81.

Endereço para correpondência:

Bárbara C. C. Silva

Rua Uberaba, 370, sala 901 - Barro Preto

30180-080, Belo Horizonte MG

Email: barbaracampolina@mac.com 